\title{
Multisystem Inflammatory Syndrome in Children (MIS-C) Associated with Novel Coronavirus (SARS-CoV-2) Infection: A Report on Two Cases
}

\author{
Begum NNF$F^{1}$, Al-Amin $\mathrm{A}^{2}$, Begum $\mathrm{M}^{3}$, Sarker MFR ${ }^{4}$, Bhuiyan $\mathrm{N}^{5}$, Khan $\mathrm{AA}^{6}$, Chowdhury $\mathrm{S}^{7}$, Ferdous $\mathrm{J}^{8}$
}

DOI: https://doi.org/10.3329/jafmc.v16i1.53847

We report two critically ill children (aged 5-8 years), presented with features of multisystem inflammatory syndrome in children (MIS-C) from January 1 to February 2, 2021 at a tertiary-care center (Combined Military Hospital) in Dhaka, Bangladesh. Both of the previously healthy children tested positive for severe acute respiratory syndrome coronavirus 2 (SARS-CoV-2) infections. Clinical presentations were similar in both with fever, gastrointestinal complaints, respiratory distress, rash, headache and myalgia. Laboratory values were high levels of $\mathrm{C}$-reactive protein, D-dimers, B-type natriuretic peptide (Pro-BNP), troponin I and low albumin levels in both patients. Evaluations for other infectious diseases werenegative. Both the patients were critically ill, requiring admission to the pediatric intensive care unit (P-ICU) due to circulatory shock and needed inotropes. One of the patients had respiratory failure and required mechanical ventilation. Both patients received steroids, Intravenous Immunoglobulin (IVIG), Remdesivir, Tocilizumab. MIS-C is a recently recognized pediatric illness spectrum in association with SARS-CoV-2 infection. As for manifestations of COVID-19 infection and its consequences in children is myriad and knowledge about MIS-C is evolving, reporting is essential for better understanding of clinical clues of MIS-C and finding out a panacea through experience sharing.

Key-words: Multisystem Inflammatory Syndrome (MIS-C), Reverse transcription-polymerase chain reaction (RT-PCR), B-type natriuretic peptide (BNP), Creatinine Kinase Myocardial Band (CKMB).

\section{Introduction}

The world is suffering through the global pandemic of coronavirus disease (COVID-19), caused by the novel coronavirus; severe acute respiratory syndrome coronavirus 2 (SARS-CoV-2). Initially, it was thought that children are less severely affected ${ }^{1-3}$; SARS-CoV-2 infection has recently been found to be associated with a novel set of clinical manifestations called multisystem inflammatory syndrome in children (MIS-C) $)^{4}$. The Centers for Disease Control and Prevention (CDC) has declared MIS-C to be a reportable illness as of May 14, 2020 and has recently provided a case definition which includes patients under 21 years of age with fever $\left(>38.0^{\circ} \mathrm{C}\right.$ for $\geq 24$ hours, or report of subjective fever lasting $\geq 24$ hours), laboratory evidence of inflammation [one or more of the following: elevated C-reactive protein (CRP), erythrocyte sedimentation rate (ESR), fibrinogen, procalcitonin, D-dimer, ferritin, lactic acid dehydrogenase (LDH), or interleukin 6 (IL-6), elevated neutrophils, reduced lymphocytes, and low albumin)], severe illness needing hospitalization and involvement of two or more organ systems (cardiac, renal, respiratory, hematologic, gastrointestinal, dermatologic, or neurological), with positive testing for SARS-CoV-2 indicating current or recent infection or COVID-19 exposure; and no other alternative plausible diagnoses ${ }^{4}$. We recently encountered many cases of MIS-C with a wide spectrum of presentation starting from mild to a critical degree. Here, we reportedtwo critically ill patients with MIS-C associated with SARS CoV-2 infections admitted to a tertiary-care center in Dhaka, Bangladesh.

\section{Case Reports}

Case-1: A 5 years 4 months old boy with no significant past medical history presented at emergency following one day of febrile illness (temp $40.6^{\circ} \mathrm{C}$ ), associated with vomiting, progressively worsening diffuse abdominal pain, and multiple episodes (4-5 times per day) of greenish, watery stools, not mixed with blood. There was an associated headache, lethargy, and myalgia but no chest pain or respiratory distress. His family members were RT-PCR positive for SARS CoV-2 four to six weeks back. On admission, he was lethargic, dehydrated, febrile with a temperature of $400 \mathrm{C}$, and had tachycardia (heart rate of 154 beats per minute). Oxygen saturation and blood pressure were within the normal limit. There was diffuse abdominal tenderness on the abdominal examination. Three days after admission, he developed hypotensive shock, urgently shifted to PICU, treated with multiple fluid boluses and inotropic support with Dopamine. Subsequently, Dobutamine and Noradrenaline were added. Laboratory investigations showed leukocytosis, neutrophilia, Iymphopenia, thrombocytopenia and hypoalbuminemia, with elevated levels of CRP, procalcitonin, ferritin, LDH, fibrinogen, FDP, D-dimers, troponin-I, Pro- BNP and CKMB. Arterial blood gas showed metabolic acidosis with lactic acidosis. Blood and urine cultures and multiplex PCR for respiratory pathogens were negative for any organism. The patient had a negative SARS-CoV-2 RT-PCR but IgG for SARS CoV-2 was

\footnotetext{
1. Brig Gen Nurun Nahar Fatema Begum, SBP, MBBS, FCPS, FRCP, FACC, FSCAI, Professor \& Head, Department of Paediatrics, AFMC \& Adviser Specialist in Paediatric Cardiology, CMH, Dhaka (E-mail: colfatema@hotmail.com) 2. Lt Col Abdullah Al-Amin, MBBS, FCPS, Classified Child Specialist, CMH, Dhaka 3. Lt Col Maksuda Begum, MBBS, DCH, Classified Child Specialist \& Hematologist, CMH, Dhaka 4. Col Md Ferdousur Rahman Sarker, MBBS, DCH, FCPS, Classified Child Specialist and Paediatric Cardiologist, CMH, Dhaka 5. Lt Col Nazmul Islam Bhuiyan, MBBS, FCPS, Classified Child Specialist and Paediatric Cardiologist, CMH, Dhaka 6. Lt Col Ashfaque Ahmmed Khan, MBBS, DCH, FCPS, Classified Child Specialist and Paediatric Cardiologist, CMH, Dhaka 7. Lt Col Sayla Chowdhury, MBBS, FCPS, Classified Child Specialist, CMH, Dhaka 8. Lt Col Jannatul Ferdous, MBBS, FCPS, Classified Child Specialist and Paediatric Cardiologist, CMH, Dhaka.
} 
Table-I: Clinical characteristics of patients

\begin{tabular}{|c|c|c|c|}
\hline \multicolumn{2}{|c|}{ Characteristics of Patients } & Patient 1 & Patient 2 \\
\hline \multicolumn{2}{|c|}{ Age } & 5 years 4 months & 8 years \\
\hline \multicolumn{2}{|c|}{ Sex } & Male & Male \\
\hline \multicolumn{2}{|c|}{ Time to presentation } & 1 day & 9 days \\
\hline \multicolumn{2}{|c|}{ SARSCoV-2 test } & RT-PCR negative, IgG positive & RT-PCR positive \\
\hline \multirow{21}{*}{ 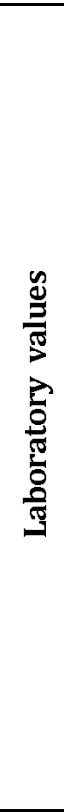 } & Leucocytes ( 4-11 X109/ L) & 22.5 & 36.9 \\
\hline & Neutrophil (\%) & $95 \%$ & $94 \%$ \\
\hline & Lymphocyte(\%) & $3 \%$ & $4 \%$ \\
\hline & Platelet ( 150-400 X109/ L) & 74 & 393 \\
\hline & C- reactive protein $(\mathrm{CRP})$ & 67 & 77 \\
\hline & Procalcitonin $(\mathrm{ng} / \mathrm{ml})$ & 72.1 & 71.79 \\
\hline & S. potassium $(\mathrm{mmol} / \mathrm{L})(3.6-5.2)$ & 2.5 & 3.8 \\
\hline & S. calcium & 6 & 7.1 \\
\hline & Fibrinogen (mg/ dL) (183-503) & 200 & 313 \\
\hline & FDP $(\mathrm{mcg} / \mathrm{ml})$ & 27 & 32.5 \\
\hline & D-dimar $(\mathrm{mcg} / \mathrm{ml})(\varangle 0.5)$ & 7.4 & 17 \\
\hline & S. Ferritin (ng/ml) (13-145) & 15000 & 17090 \\
\hline & Albumin $(\mathrm{gm} / \mathrm{dL})(3.8-5.4)$ & 2.8 & 2.3 \\
\hline & S. creatinine $(\mathrm{mg} / \mathrm{dL})(0.6-1.3)$ & 0.5 & 0.6 \\
\hline & $\mathrm{LDH}(\mathrm{U} / \mathrm{L})$ & 6921 & 4349 \\
\hline & Troponin I (ng/ml) & 145 & 157 \\
\hline & Pro-BNP(B-type natriuretic peptide) $\mathrm{pg} / \mathrm{ml}$ & 4012 & $>35,000$ \\
\hline & $\mathrm{CPK}(\mathrm{U} / \mathrm{L})$ & 5018 & 2749 \\
\hline & S. amylase (U/L) $(<220)$ & 441 & 459 \\
\hline & S. lipase (U/ L) $(<160)$ & 211 & 1329 \\
\hline & Chest X-ray & Bilateral pleural effusion & Mild pleural effusion on left side \\
\hline \multirow{6}{*}{\multicolumn{2}{|c|}{ Echocardiogram }} & LVEF-45\% & Poor LV function \\
\hline & & FS:22\% & LVEF $40 \%$ \\
\hline & & CORONARIES: dilated & FS 20\% \\
\hline & & LMCA:9.3mm & Coronaries: normal \\
\hline & & LAD $>2.5 \mathrm{~mm}$ & \\
\hline & & Borderline LV function & \\
\hline \multirow{2}{*}{\multicolumn{2}{|c|}{$\begin{array}{l}\text { USG of the whole abdomen } \\
\text { Duration of ICU stay (days) }\end{array}$}} & Moderate ascites & Mild ascites \\
\hline & & 11 & 13 \\
\hline
\end{tabular}

positive. Clinical features and laboratory values are summarized in Table-I. An echo- cardiogram showed a left ventricular ejection fraction of $45 \%$ with a shortening fraction of $22 \%$ which is significantly below the normal level. On hospital stay, he developed cracked lips and palmer edema. He also developed ascites and bilateral pleural effusion in course of the disease.

Following a multidisciplinary approach, a board of specialists was formed including a pediatric cardiologist, pulmonologist, gastroenterologist, rheumatologist, nephrologist and intensive care specialist to treat the child. He received intravenous antibiotics (Piperacillin and Tazobactam combination and linezolid). He received IVIG (intravenous immunoglobulin at 2 $\mathrm{g} / \mathrm{Kg}$ ), methylprednisolone, Remdesivir, Tocilizumab and Enoxaparin/ Heparin for treatment. He became afebrile on day 7 of hospitalization. He was discharged home after 11 days of hospital stay with oral anti-coagulant (Rivaroxaban) and diuretic for 2 more weeks, anti-platelet (Aspirin) for 6 months and a follow-up visit plan in pediatric cardiology, rheumatology, and gastroenterology OPD clinic in a week, and echocardiogram after 1 month. Follow-up visit on cardiology OPD showed no residual cardiac abnormality after one month.
Case-2: An 8 years old boy with no significant past medical history heli-evacuated from Chattogram presented with a fever up to $39.5^{\circ} \mathrm{C}$ for nine days with associated headaches, lethargy, myalgia, vomiting, abdominal pain, and diarrhea. On admission, he had a temperature of $40^{\circ} \mathrm{C}$, heart rate of 138 beats per minute, respiratory rate of 24 breaths per minute, and oxygen saturation of $100 \%$ on room air. Two hours later, he was hypotensive with a blood pressure of $70 / 40 \mathrm{~mm} \mathrm{Hg}$ with a weak pulse requiring multiple fluid boluses and inotrope support and developed respiratory failure needing intubation and mechanical ventilation. The patient was noted to have leukocytosis, neutrophilia, lymphopenia, thrombocytopenia and hypo- albuminemia, elevated levels of inflammatory markers like CRP, Procalcitonin, Pro-BNP, D-dimers, ferritin, and troponin I (Table-I). The patient had a positive RT PCR for SARS-CoV-2. During the hospital stay, he further developed right-sided pleural effusion and mild ascites. He also developed palmer erythema, cracked lips, and later peeling of periungual skin.

A multidisciplinary approach was instituted. The patient received broad-spectrum intravenous antibiotics (Meropenem and Linezolid), IVIG (2g/kg), Methylprednisolone, Remdesivir, Tocilizumab and 
Enoxaparin. He became afebrile on the 4th day of hospitalization. He was extubated on day 9 of intubation and gradually weaned off oxygen by another 7 days. He was discharged after a hospital stay of 17 days with a similar follow-up plan. Follow-up visit on cardiology OPD showed no residual cardiac abnormality after one month.

\section{Discussion}

We have been treating MIS-C in our hospital since April 2020, which was initially labeled as Kawasaki-like disease or Macrophase Activation Syndrome and treated with IVIG before it was labeled as MIS-C by CDC. This is the first report of MIS-C associated with SARS-CoV-2 infection in $\mathrm{CMH}$, Dhaka in two hospitalized patients from January 1 to February 2, 2021, who (aged 5-8 years) were proven by the presence of IgG antibody against SARS-Cov-2 in one case and RT-PCR positive in another case. They developed systemic inflammation followed by multi-organ involvement leading to critical illness necessitating pediatric intensive care.

The condition is being recently recognized; so, very few articles published so far in Bangladesh. When we first received these patients, we were thinking about MIS-C as the pandemic is still prevailing, so we sent all the necessary investigations needed to exclude MIS-C. To our utter surprise, all the investigation reports came out supporting MIS-C. While awaiting reports, we were treating the cases as per our initial differential diagnoses like acute gastroenteritis in the first case and enteric fever in the second with symptomatic and antibiotics. We followed the American College Rheumatology(ACR) guideline for MIS- $\mathrm{C}^{5}$ for treatment. As the disease evolved we distributed the medication and supportive care in the following headings: anti-Inflammatory (Steroid, high dose aspirin), anti- coagulants (heparin, rivaroxaban), anti failure (Carvedilol, furosemide, Digoxin, Ivabradine) antiplatelet (low dose Aspirin) antibiotics, biological agents (IVIG, Tocilizumab, Anakinra), fluid and electrolytes, intake output balance, nutrition, cardiac monitoring (Coronary care), counseling etc.

In course of the disease, both of our patients developed ascites and pleural effusion that gave an impression somewhat like Dengue fever which is also endemic in Bangladesh. That thought led us to fluid management like Dengue fever and subsequently Dengue fever was excluded by investigation. It was a different experience in our cases. We have treated ascites and pleural effusion with low dose Furosemide (1mg/kg over 24 hours i/v infusion) along with Dopamine, occasional fresh frozen plasma, and albumin infusion (colloids) intending to enforce the diuresis rather than leaking of plasma to third space. This protocol of encouraging preferential renal flow was found very effective in our cases in reducing plasma leakage effectively.

A cohort study in UK ${ }^{6}$ found Children with MIS- $C$ were five times more likely to be admitted to critical care (73\%), in this case both the patient needed Critical Care. Children meeting MIS-C criteria were older (median age 10.7 (8.3-14.1yr), in this case age were between 5-8 years. In addition to the WHO criteria, children with MIS-C were more likely to present with fatigue (51\%), headache (34\%), myalgia (34\%) sore throat (30\%) and lymphadenopathy. In this case, both the patient had fatigue, headache and myalgia but no sore throat or lymphadenopathy $y^{7,8}$.

Another study showed elevated levels of C-reactive protein, d-dimer and troponin in $100 \%, 91 \%$ and $71 \%$ of the patients, respectively; $62 \%$ received vasopressor support, $53 \%$ had evidence of myocarditis, $80 \%$ were admitted to an intensive care unit ${ }^{9}, 10$. Cardiac manifestations, including coronary and myocardial involvement, are common and should be carefully identified ${ }^{10}$. Both the patient in this report developed left ventricular failure (LVF) following myocarditis and had elevated CRP, d-dimer, and troponin. In the first case, there was dilated coronary artery also. Patients were treated with IVIG, Methylprednisolone, Remdesivir which matches with other hospitals treating MIS- $C^{9-11}$, but they could introduce Aspirin at an anti- inflammatory dose at the onset which we could not due to gastrointestinal problem.

One of these patients had thrombocytopenia. A Cohort Study in $\mathrm{UK}^{6}$ found $32 \%$ of patients with thrombocytopenia. Clinical presentation of MIS-C with multi-organ involvement and elevated inflammatory markers may have overlapping features with Kawasaki Disease, Macrophage Activation Syndrome and Toxic Shock Syndrome, but MIS-C presently understood to be a separate phenomenon ${ }^{9-11}$. MIS-C is thought to be related to a post-viral immune-mediated inflammatory process, as suggested by recognition of clinical cases as we move into the post-peak phase of COVID-19 illness incidence, although the pathogenesis of the syndrome remains largely unclear ${ }^{4}$.

Early recognition is critical because the patient can deteriorate quickly. We had an experience of losing a child because of delayed arrival at the tertiary care facility. We have treated our cases in a resource constraint set up with utmost care and whatever medicines available in our setup.

\section{Conclusion}

We observed two critically ill multisystem inflammatory syndromes in children (MIS-C) with SARS-CoV-2 infection who were previously healthy. In our cases, the diagnosis was early, even before the full-blown sign and symptoms of the disease. Early diagnosis $^{9}$ and management in time lead to a successful story in both cases. As manifestations of COVID-19 in children is myriad and knowledge about MIS-C is evolving, reporting is essential for a better understanding of clinical clues of MIS-C and finding out a panacea through experience sharing.

\section{References}

1. Kest H, Kaushik A, DeBruin W et al. Multisystem Inflammatory Syndrome in Children (MIS-C) Associated with 2019 Novel Coronavirus (SARS-CoV-2) Infection. Case Reports in Pediatrics 2020:1-4. 
2. Parvin R, Afroze $S$, Bashar AHMK et al. Neonate with Rare Clinical Presentation of COVID-19: A Report of Two Clinically Distinct Cases. Bangladesh J Infect Dis 2020; 7(2):102-6.

3. CDC COVID-19 Response Team. Coronavirus disease 2019 in children-United States. Morbidity and Mortality Weekly Report (MMWR) 2020; 69(14):422-6.

4. Centers for Disease Control and Prevention (CDC). Health advisory on the multisystem inflammatory syndrome in children (MIS-C) associated with coronavirus disease 2019". Avaialable at https://emergency.cdc.gov/ han /2020/han00432.asp?

5. Handerson LA, Canna SW, Friedman KG. American College of Rheumatology Clinical Guidance for multisystem Inflammatory Syndrome in children (MIS-C) Associated with SARS-COV-2 and Hyper inflammation in COVID-19: version-2. Arthritis \& Rheumatology 2021; 73(4):e13-e29.

6. Swann OV, Holden KA, Turtle Let al. Clinical characteristics of children and young people admitted to hospital with COVID-19 in the United
Kingdom: Prospective multi-center observational cohort study. BMJ 2020; 370:m3249

7. Fatema NN, Nazme NI, AKhan AA et al. COVID-19 Pandemic: A focused review on pediatric patients. Cardiovasc J 2020; 13(1):62-74.

8. NN Fatema, NI Nazme. Multisystem Inflammatory syndrome in children (MIS-C): A brief review. CMHDJ Bangladesh 2021; 2(1):5-8.

9. Mahajan N, Chang HT, Leeman R et al. Case of a multisystem inflammatory syndrome in children presenting as fever and abdominal pain. BMJ Case Rep 2020; 13:e237306.

10. Dufort EM, Koumans EH, Chow EJ et al. New York State and Centers for Disease Control and Prevention Multisystem Inflammatory Syndrome in Children Investigation Team. Multisystem Inflammatory Syndrome in Children in New York State. N Engl J Med 2020; 383(4):347-58.

11. Ghodsi A, Mahmoudabadi E, Ghahremani S et al. Cardiac Manifestations of Multisystem Inflammatory Syndrome in Children (MIS-C) Associated with SARS-CoV-2 Infection. Arch Pediatr Infect Dis 2021:e109915 